\title{
Simulated Rotor Wake Interactions Resulting from Civil Tiltrotor Aircraft Operations Near Vertiport Terminals
}

\author{
Larry A. Young ${ }^{1}$ \\ NASA Ames Research Center, Moffett Field, CA, 94035 \\ Ganesh Rajagopalan ${ }^{2}$ \\ Sukra-Helitek, Inc. and Iowa State University, Ames, IA 50011
}

\begin{abstract}
A mid-fidelity computational fluid dynamics tool called RotCFD - specifically developed to aid in rotorcraft conceptual design efforts - has been applied to the study of rotor wake interactions of civil tiltrotor aircraft in the immediate vicinity of vertiport/airport ground infrastructure. This issue has grown in importance as previous NASA studies have suggested that civil tiltrotor aircraft can potentially have a significant impact on commercial transport aviation. Current NASA reference designs for such civil tiltrotor aircraft are focused on a size category of 90-120 passengers. Notional concepts of operations include simultaneous non-interfering flight into and out of congested airports having vertiports, that is, prepared VTOL takeoff and landing zones, or underutilized short runways for STOL operation. Such large gross-weight vehicles will be generating very high induced velocities. Inevitably, the interaction of the rotor wake with ground infrastructure such as terminals/jetways must be considered both from an operational as well as design perspective.
\end{abstract}

\section{Nomenclature}

$C_{p} \quad=$ pressure coefficient

$C_{T} \quad=$ rotor thrust coefficient

$\mathrm{h} / \mathrm{R}=$ ratio of rotor height-above-ground with respect to rotor radius

$v_{w} \quad=$ wind speed

$\mathrm{x} / \mathrm{R}=$ ratio of rotor horizontal-distance from vertical wall/plane, or correspondingly building, to rotor radius

$\Delta \varphi \quad=$ relative angular orientation of rotors to vertical wall/plane, or correspondingly building; with zero deg. orientation, the rotors are parallel to the wall/building.

$\Delta \psi \quad=\quad$ relative direction of wind with respect to aircraft; zero deg. is towards the aircraft nose.

\section{Introduction}

$I^{\mathrm{T}}$ has long been envisioned that rotorcraft in general, and tiltrotor aircraft in particular, might one day provide a significant role in commercial transport aviation. Several NASA-sponsored studies over the past two decades have reinforced this vision of commercial transport rotorcraft. Recent studies, Refs. 1-3, have focused on tiltrotor aircraft in the size category of 90-120 passengers. Such studies have shown that large civil tiltrotors can have a significant influence on reducing National Airspace System (NAS) delays and increasing throughput. To accomplish such gains it will be necessary to either develop and make use of vertiports (prepared VTOL takeoff and landing zones) and short underutilized runways at congested airports. Figure 1 is a simple illustration of a notional airport layout in which there is both a vertiport terminal and short runway for tiltrotor operations. A major consideration in the development of vertiports co-located at airports is the magnitude of rotor/wake interactions (i.e. in-ground-effect groundwash) from such large vehicles in close proximity to ground infrastructure.

\footnotetext{
${ }^{1}$ Aerospace Engineer, Aeromechanics Branch, Flight Vehicle Research and Technology Division, Mail Stop 24312, AIAA Associate Fellow.

${ }^{2}$ Professor, Department of Aerospace Engineering.
} 


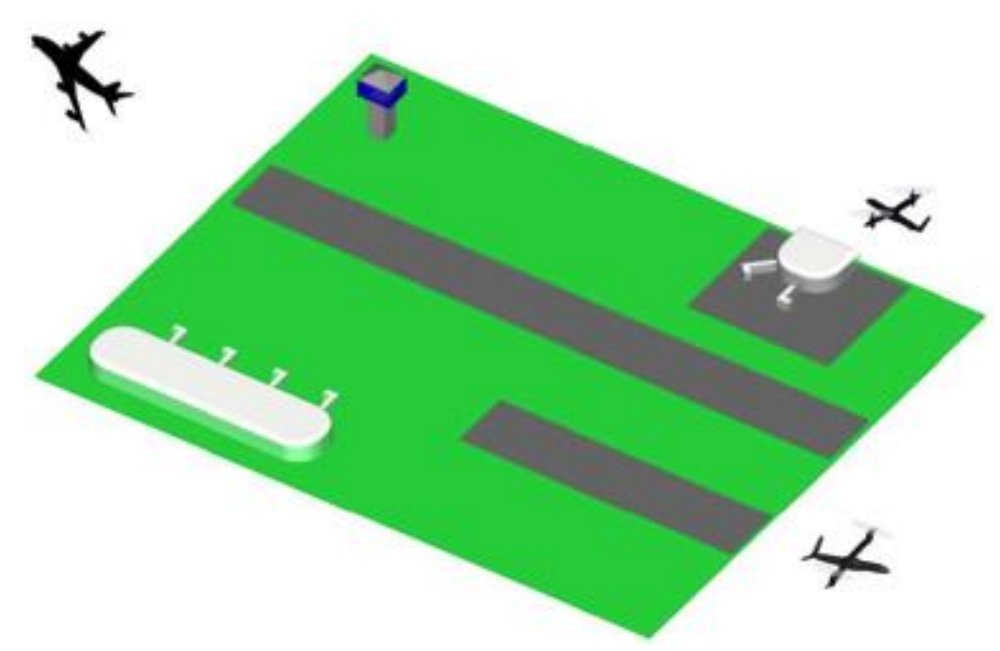

Figure 1. On-Airport-Property Vertiport Tiltrotor Operations (VTOL and STOL).

\section{Scope of the Problem}

In order to assess the compatibility of the NASA civil tiltrotor (CTR) reference designs with the anticipated Concept of Operations (CONOPS) of such aircraft operating into and out of major congested airports (circa 2025) it is necessary to use computational fluid dynamics (CFD) tools to predict rotor/wake interactions, particularly rotor groundwash, in the immediate vertiport/terminal area. The CFD tool of choice for this study is RotCFD. RotCFD is a mid-fidelity CFD tool aimed at conceptual design. The technical approach for this study is to begin with simple rotor in-ground-effect (IGE) predictions, then proceed to dual side-by-side rotor IGE predictions, followed by a complete LCTR2 aircraft (Ref. 6) IGE predictions, followed by introducing walls, planes and simple geometric shapes representing buildings, and then concluding with a LCTR2 aircraft operating near a more realistic modeling of a vertiport terminal facility. Figure 2 and 3 are representative RotCFD results for the LCTR2 reference design operating near a vertiport terminal building.

In parallel with the above set of predictions, RotCFD will be validated for this class of problem. The singleand dual-rotor IGE predictions can be compared to analytical results, e.g. Refs. 7, 9, 10, and to data from experimental studies similar to Ref. 8 . 


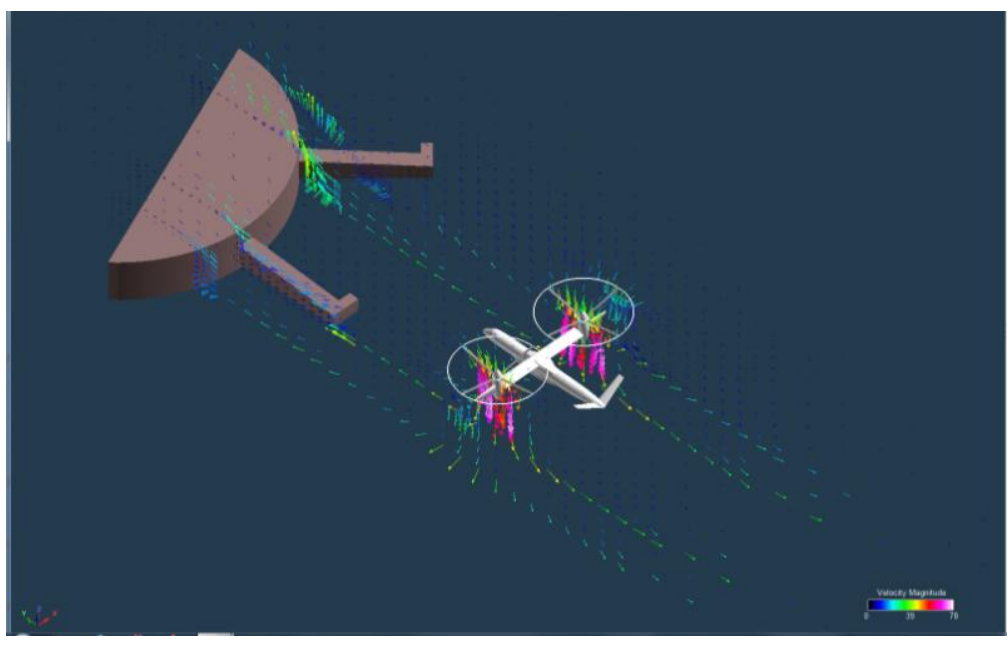

Figure 2. Representative Rotor/Wake Interaction for Civil Tiltrotor in vicinity of Vertiport (Velocity Vectors)

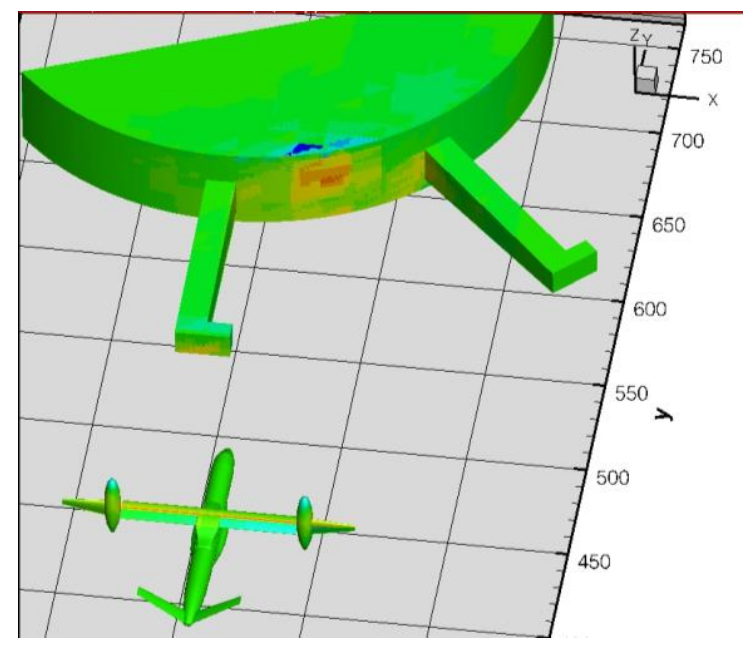

Figure 3. Representative Rotor/Wake Interaction for Civil Tiltrotor in vicinity of Vertiport (Surface Pressures on Aircraft and Building)

\section{Description of Analysis Approach}

\section{A. RotCFD Tool}

RotCFD attempts to bridge the two worlds of design and Computational Fluid Dynamics (CFD) with the help of an Integrated Design Environment (IDE) specific to rotorcraft. RotCFD emphasizes user-friendliness and efficiency that streamline the design process from geometry to CFD solution. The key components of RotCFD are a geometry module, a semi-automated grid generation module, a flow-solver module, a rotor module, a flow visualization and analysis module, all integrated in one environment. The concept of rotor blades represented by momentum sources forms the basis of this aerodynamic simulation tool for rotorcraft. The rotor momentum sources are primarily a function of the local velocity of the flow and the two-dimensional airfoil characteristics of the rotor blades. The Navier-Stokes equations and the blade element theory are coupled implicitly to yield a self-contained method for 
generating performance, as well as the near and far wake including all the aerodynamic interference inherent in a situation.

During initial design studies, parametric variation of vehicle geometry is routine. A simple geometry tool (modest CAD functionality) is offered to simplify geometry manipulation. The current version of RotCFD can read the body geometry in STL or Plot3D format. Also, some simple shapes (bodies of revolution) can be generated from within RotCFD. In addition to general transformations such as translation, rotation and scaling, parametric variation of the geometry is available to assist with the design. Graphical visualization is used to make the geometry manipulation user-friendly: the user is able to see a preview of the geometry change before committing the change.

The RotCFD Cartesian unstructured (hanging node) grid is economical to generate and can be completely automated. The cells that intersect the body, and all the cells that immediately surround the intersected cells are replaced by tetrahedra. The simple shape of the tetrahedron allows the grid to be morphed slightly in order to conform to the body. Thus the body conforming grid generator modifies a Cartesian octree grid (Cartesian here meaning all faces are aligned with the Cartesian planes) such that the grid will approximately conform to the surface of the body. The grid is approximate since the algorithm only considers the intersections between the surface geometry and the edges of the original grid. New surface faces are generated based on those edge-surface intersections instead of using the original polygons from the surface geometry. This approximate approach avoids the technical difficulties associated with maintaining the surface geometry exactly as it intersects the grid, and allows the development of a simpler and more robust algorithm with a much shorter development time. The approach also has the advantage that clean geometries are not required, only that they be closed surfaces.

The flow field of a rotor is complex. Even an isolated rotor is dominated by the mutual, aerodynamic interference effects of the blades. Therefore, all simulation techniques, to be successful, must consider interference effects. Any numerical algorithm that solves the Navier-Stokes equations is adequate for obtaining the flow field. The SIMPLER/SIMPLE (Ref. 4) line of pressure-based algorithms is suitable for low speed flows and currently SIMPLE is used for solving the flow field in RotCFD. Additional details describing RotCFD are summarized in Ref. 5.

\section{B. The LCTR2 Reference Design Vehicle}

The baseline vehicle design used in this study is a NASA reference design described in Ref. 6 . The LCTR2 is a 90-passenger vehicle and has been used in many past studies. Alternate large civil tiltrotor vehicle conceptual designs are available, e.g. the vehicle designs detailed in Refs. 1-3, but the LCTR2 reference design is perhaps the most well-known. The RotCFD models are based on LCTR2 CAD geometry files that have been recently used to fabricate small-scale wind tunnel models of the LCTR2 vehicle.

\section{Notional Terminal/Vertiport Layouts}

The principal guide for vertiport design used in this study is Ref. 11. This FAA Advisory Circular, Ref. 11, has subsequently been canceled since its initial release in the mid-90's, but still represents the best source of vertiport design guidance available. The notional vertiport layout illustrated in Fig. 2-3 is somewhat inspired by certain terminals at Newark Liberty International airport. However, one of the key challenges of this study is to derive general results for rotor/wake interactions for CTR operating near vertiports and other airport ground infrastructure that are not unduly biased by the models used for such ground infrastructure.

\section{Preliminary Results for Vertiport Problem}

Figure 4a-b illustrates one partial sweep of the LCTR2 aircraft, near a notional vertiport terminal, where the height above ground of the vehicle is varied. In Fig. 4, the LCTR2 vehicle is initially in ground effect and then rises out of ground effect as the vehicle height increase. In the cases where the vehicle is in IGE the rotor groundwash significantly interacts with the vertiport terminal building. Key metrics in the final paper will be the location, direction, and magnitude of the maximum groundwash velocities predicted as well as, in the cases where vertiport buildings are modeled, the maximum and minimum surface pressures predicted on such notional buildings. 

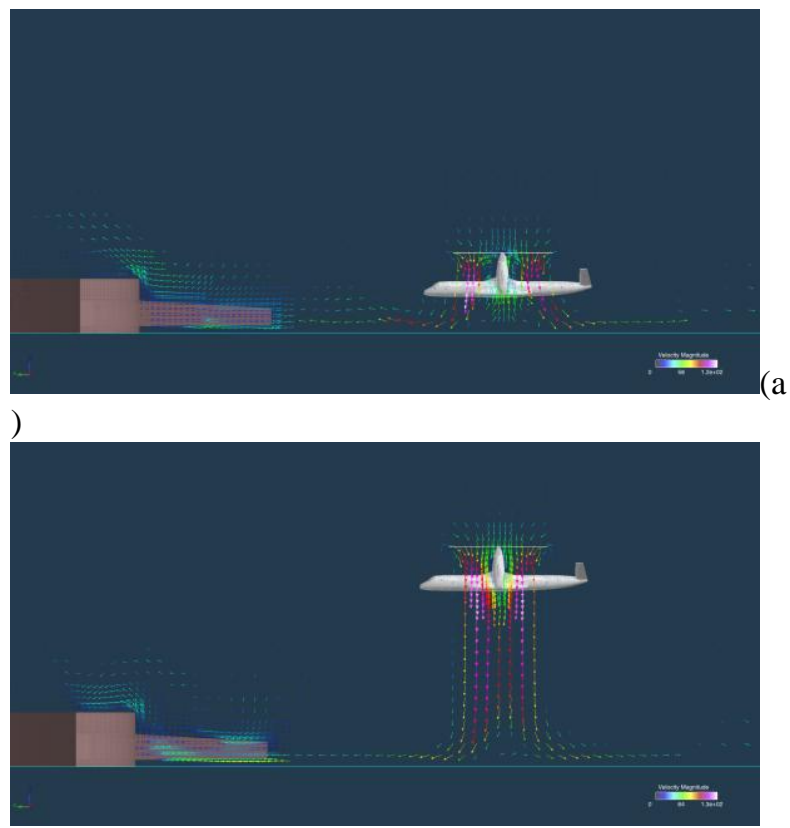

(b)

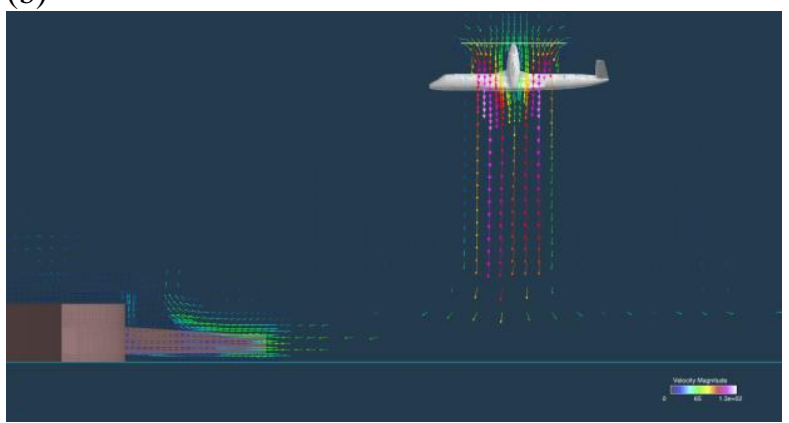

(c)

Figure 4. Height Sweep of LCTR2: (a) $h / R=1.39$; (b) $h / R=4.16 ; h / R=5.70$.

The ultimate outcome of this study is anticipated to be threefold. First, the information gleaned from this study will help refine future NASA reference designs for civil tiltrotor aircraft. Second, the results from this study might help influence future vertiport design considerations. Third, the results might also influence the concepts of operation of such vehicles in the airport environment such that the efficiency and safety of CTR operations at vertiports can be successfully balanced.

\section{Concluding Remarks}

A mid-fidelity computational fluid dynamics tool, one especially tailored for conceptual design studies of rotarywing vehicles, is being applied to the problem of rotor/wake interactions (principally groundwash) in the vertiport environment. The rotor induced velocities from very large gross weight vehicles can be potentially hazardous and must be better understood. The results from this study are anticipated to help guide future NASA studies of the introduction of this new class of vehicles into the National Airspace System. 


\section{Acknowledgments}

This work was supported in part by the Subsonic Rotary Wing project of the Fundamental Aeronautics Program of the NASA Aeronautics Research Mission Directorate; the work was also supported in part by the NASA SBIR program.

\section{References}

${ }^{1}$ Young, L.A., et al, "Civil Tiltrotor Aircraft Operations," 11th AIAA Aviation Technology, Integration, and Operations (ATIO) Conference, Virginia Beach, VA, Sept. 20-22, 2011.

${ }^{2}$ Young. L.A., et al, "A Study of Civil Tiltrotor Aircraft in NextGen Airspace," AIAA-2010-9106, 10th AIAA Aviation Technology. Integration, and Operations (ATIO) Conference, Fort Worth, TX, Sept. 13-15, 2010.

${ }^{3}$ Chung, W.W., et al, "Modeling High-Speed Civil Tiltrotor Transports in the Next Generation Airspace," NASA CR 2011-215960, October 2011.

${ }^{4}$ Patankar, S.V., Numerical Heat Transfer and Fluid Flow, Hemisphere Publishing Corp, New York, 1980.

${ }^{5}$ Rajagopalan, G., "RotCFD a Tool for Aerodynamic Interference of Rotors: Validation and Capabilities," Future Vertical Lift Aircraft Design Conference, San Francisco, CA, January 18-20, 2012.

${ }^{6}$ Acree, C.W., Yeo H. and Sinsay, J.D., "Performance Optimization of the NASA Large Civil Tiltrotor," Joint AHS/AIAA/SAE/RAeS International Powered Lift Conference (IPLC), London, UK, July 22-24, 2008.

${ }^{7}$ Young, L.A. and Derby, M.R., "Rotor/Wing Interactions in Hover," NASA TM 2002-211392, April 2002.

${ }^{8}$ Martin, P., "Tiltrotor/Building Interaction Test: 7x10 Wind Tunnel," Private Communication/Unpublished Data, May 2011.

${ }^{9}$ Rossow, V., "Effect of Ground and Ceiling Planes on Thrust of Rotors in Hover," NASA TM 86754, July 1985.

${ }^{10}$ Johnson, W., Helicopter Theory, Princeton University Press, 1980, pp. 122-124.

${ }^{11}$ FAA Advisory Circular, "Vertiport Design," AC \# 150/5390-3, May 31, 1991. 